\title{
Block Third Derivative Trigonometrically-Fitted Methods for Stiff and Periodic Problems
}

\author{
R. I. Abdulganiy ${ }^{\mathrm{a}, *}$, O. A. Akinfenwa ${ }^{\mathrm{b}}$, O. A. Yusuff ${ }^{\mathrm{b}}$, O. E. Enobabor ${ }^{\mathrm{c}}$, S. A. Okunuga ${ }^{\mathrm{b}}$ \\ ${ }^{a}$ Distance Learning institute, University of Lagos, Nigeria \\ ${ }^{b}$ Department of Mathematics, University of Lagos, Nigeria \\ ${ }^{c}$ Department of Mathematics, Yaba College of Technology, Lagos, Nigeria
}

\begin{abstract}
This article constructed and implemented a family of a third derivative trigonometric fitted method of order $k+3$ whose coefficients are functions of frequency and step size for the integration of systems of first-order stiff and periodic Initial Value Problems. The Block Third Derivative Trigonometric Fitted methods (BTDTFMs) are constructed via multistep collocation technique and applied in block form as simultaneous numerical integrators which make them self-starting. The basic properties of the BTDTFMs are analyzed and presented. The accuracy and efficiency of the methods based on number of function evaluation are established through some standard numerical examples which are either stiff or periodic in nature.
\end{abstract}

Keywords: Convergence, Frequency, Stiff, Trigonometrically-Fitted.

Article History :

Received: 02 January 2020

Received in revised form: 16 February 2020

Accepted for publication: 17 February 2020

Published: 28 February 2020

(C)2020 Journal of the Nigerian Society of Physical Sciences. All rights reserved. Communicated by: T. Latunde

\section{Introduction}

A-stable methods are of great importance in solving stiff problems but put stark limitations on the choice of suitable methods for stiff problems. Dahlquist [1] established that the most accurate A-stable linear multistep method has order 2. The search for higher-order A-stable multistep method is carried out in the following three major directions (Biala et al. [2]):

1. Use of higher derivatives of the solution;

2. Incorporating additional stages and hybrid points;

\footnotetext{
${ }^{*}$ Corresponding author tel. no: +2348035195870

Email addresses: profabdulcalculus@gmail .com (R. I. Abdulganiy), akinolu35@yahoo.com (O. A. Akinfenwa),

olaoluwa. yusuff@gmail . com (O. A. Yusuff),

enobaborosaretin@yahoo.com (O. E. Enobabor),

waleokunuga@gmail.com (S. A. Okunuga)
}

3. Incorporating super future points.

Many numerical methods have been developed along these three directions which include but not limited to Gear [3], Enright [4], Cash [5], Wu [6], Hojjati [7], Jator [8], Sahi et al. [9], Ngwane and Jator [10], Akinfenwa et al. [11,12,13], Mehdizadeh et al [14] and Abdulganiy et al. [15].

Method based on Simpson's was explored by Akinfenwa et al. [16] while methods bases on exponential fitting were considered in Okunuga [17], Vaquero and Vigo-Aguiar [18], Abhulimen et al. [19,20], Ehigie et al. [21] and Adesanya et al. [22] to numerically approximate stiff problems. Although the exponential fitting methods for solving stiff problems were easy to implement, tedious mathematical analyses are involved in obtaining the A-stability.

Periodic problems, on the other hand, have received much attention in the past few decades. The methods for solving peri- 
odic problems according to Yakubu et al., [23] can be classified into two: methods with constant coefficients and methods having coefficients depending on the frequency of the problems. If a good estimate of the frequency or some suitable substitute is known in advance, a linear multistep method can be used for the integration of periodic problems (Vanden Berghe et al., [24] and Van de Vyver [25]). The idea of using basis function which integrates a set of linearly independent function exactly other than polynomial can be traced to the work of Gautchi [26] and Lyche [27]. Many of such extension has been discussed in Coleman and Duxbury [28], Ixaru et al. [29], Vanden Berghe and his collaborators ([24], [30,31]), Simos [32,33], Tsitouras and Simos [34], Nguyen et al. [35], Senu et al. [36], Jator and his collaborators [37-41], Jator [42] and Abdulganiy and his collaborators [43-45].

In what follows, a Continuous third Derivative Method with Trigonometric Coefficients (CTDMTC) is derived via a multistep collocation technique for which the approximate interpolating function is a linear combination of polynomial and trigonometric terms. The CTDMTC is used to generate a family of Third Derivative Trigonometrically-Fitted Method (TDTFM) and some other discrete methods as by-products that are combined together into a Block Third Derivative TrigonometricallyFitted Method (BTDTFM). The BTDTFM is applied as a simultaneous numerical integrator for the integration of the system of first-order Ordinary Differential Equations (ODEs)

$$
y^{\prime}(t)=f(t, y(t)), y\left(t_{0}\right)=y_{0}, t \in\left[t_{0}, t_{N}\right]
$$

where $f$ satisfies the Lipschitz condition with a periodic solution whose frequency is known in advance. Stiff or/and periodic differential equation of the form in equation (1) frequently arises in many area of Science and Engineering, a list of such are provided in Jator and Agyingi [46], Varden Berghe et al. [24] and Ndukum et al. [47]. The methods in this paper is different from the methods in Jator [48,49] and Akinfenwa [50] in that they are used to solve system of first-order ODEs while the methods in Jator $[48,49]$ are direct numerical integrators of general second-order ODEs and the methods in Akinfenwa [50] are hybrid in nature respectively.

Some of the advantages of continuous methods include but not restricted to providing defect control (Enright [51]) and ability to produce complementary methods which are combined and applied in block-by-block fashion (Onumanyi et al. [52], Akinfenwa et al. [11,13]). The foremost block methods are credited to Milne [53], Rosser [54], Shampine and Watts [55] and Chu and Hamilton [56] and are implemented in predictorcorrector modes that reduce the stability properties of the methods. The block method in this paper is implemented without the use of predictors. According to Jator and Agyingi [46], for a block method, it is needless to make a function evaluation at the preliminary part of the original block since at all blocks (with exception of the first block) the first function evaluation is previously known from the previous block.

The rest of this paper is arranged as follows: the theoretical and basic elements of the BTDTFM is discussed in section 2 . The basic properties of the methods are discussed in section 3 .
In section 4, the estimation of the computational frequency is discussed while numerical experiments are presented in section 5. Finally, section 6 concludes the paper.

\section{Theoretical and Basic Elements of the BTDTFM}

The $k$-step third derivative method has the form

$$
y_{n+k}-y_{n+k-1}=h \sum_{j=0}^{k} \beta_{j}(u) f_{n+j}+h^{2} \delta_{k}(u) g_{n+k}+h^{3} \gamma_{k}(u) l_{n+k}(2)
$$

where $u=\omega h, \omega$ is the frequency, $\beta_{j}, \delta_{k}, \gamma_{k}$ which depends on frequency and step size are parameters to be determined uniquely. Also, $y_{n+k}$ is the numerical approximation to exact solution $y\left(x_{n+k}\right)$ and $f_{n+j}=f\left(x_{n+j}, y_{n+j}\right)$,

$$
\begin{gathered}
g_{n+k}=\left.\frac{d f(x, y(x))}{d x}\right|_{\begin{array}{l}
x \\
y \\
=y_{n+k}
\end{array}} y_{n+k} \\
l_{n+k}=\left.\frac{d^{2} f(x, y(x))}{d x^{2}}\right|_{\begin{array}{l}
x \\
y
\end{array}}=x_{n+k}
\end{gathered}
$$

In order to obtain (2), the exact solution $y(x)$ is approximated on the interval $\left[x_{n}, x_{n+k}\right]$ by the interpolating function $I(x)$ given by

$$
I(x)=\sum_{j=0}^{k+1} a_{j} x^{j}+a_{k+2} \sin (\omega x)+a_{k+3} \cos (\omega x)
$$

Imposing conditions in equations (4) and equation (4) below on equation (3)

$$
\begin{aligned}
& I\left(x_{n+j}\right)=y_{n+j} \\
& \begin{cases}I^{\prime}\left(x_{n+j}\right)=f_{n+j} & j=0(1) k \\
I^{\prime \prime}\left(x_{n+j}\right)=g_{n+j} & j=k \\
I^{\prime \prime \prime}\left(x_{n+j}\right)=l_{n+j} & j=k\end{cases}
\end{aligned}
$$

lead to a system of $(k+4)$ equations which is solved using a Computer Algebra System (CAS) such as Maple. The derivation of the methods manually becomes more difficult for $k \geq 2$. Hence, the explorations of CAS become necessary. The continuous form is developed by substituting the values of $a_{j}, \quad j=$ $0(1)(k+3)$ into equation equation (3). After some algebraic manipulations, the continuous form is obtained as the expression equation (6).

$$
I(x)=y_{n+k-1}+h \sum_{j=0}^{k} \beta_{j}(u, x) f_{n+j}+h^{2} \delta_{k}(u, x) g_{n+k}+h^{3} \gamma_{k}(u, x) l_{n+k}(6)
$$

The continuous method in equation (6) is evaluated at $x=x_{n+k}$ to generate the principal method given by 
$y_{n+k}=y_{n+k-1}+h \sum_{j=0}^{k} \beta_{j}(\cos (u), \sin (u)) f_{n+j}+h^{2} \delta_{k}(\cos (u), \sin (u)) g_{n+k}+h^{3} \gamma_{k}(\cos (u), \sin (u)) l_{n+k}$

while the $(k-1)$ secondary methods are obtained as

$$
y_{n+i}=y_{n+k-1}+h \sum_{j=0}^{k} \overline{\beta_{j}}(\cos (u), \sin (u)) f_{n+j}+h^{2} \overline{\delta_{k}}(\cos (u), \sin (u)) g_{n+k}+h^{3} \overline{\gamma_{k}}(\cos (u), \sin (u)) l_{n+k}
$$

by evaluating equation (6) at $x=x_{n+i}, i=1,2, \ldots, k-1$.

Following the steps discussed above, the secondary and principal methods for $k=2$ and $k=3$, their coefficients and the corresponding power series conversion up to $O\left(u^{10}\right)$ are given as follows For $k=2$,

$$
\begin{aligned}
& y_{n}=y_{n+1}+\overline{h \beta_{0,1}(\cos (u), \sin (u))} f_{n}+\overline{h \beta_{1,1}(\cos (u), \sin (u))} f_{n+1} \\
& +\overline{h^{2} \delta_{2,1}(\cos (u), \sin (u))} g_{n+1}+\overline{h^{3} \gamma_{2,1}(\cos (u), \sin (u))} l_{n+1} \\
& y_{n+2}=y_{n+1}+h \beta_{0}(\cos (u), \sin (u)) f_{n}+h \beta_{1}(\cos (u), \sin (u)) f_{n+1}+h \beta_{2}(\cos (u), \sin (u)) f_{n+2} \\
& +h^{2} \delta_{2}(\cos (u), \sin (u)) g_{n+2}+h^{3} \gamma_{2}(\cos (u), \sin (u)) l_{n+2} \\
& \left(\overline{\beta_{0,1}}=\frac{1}{6}\left(-14 \sin (u) u^{3}+5 u^{4}-12 \cos (u) u^{2}-6 \cos (2 u) u^{2}+12 \sin (2 u) u+6 u^{2}-24 \cos (u)+12 \cos (2 u)+12\right)\right. \\
& /\left(\left(-2 u-2 \cos (2 u) u-\sin (2 u) u^{2}-4 \sin (u)+2 \sin (2 u)+4 \cos (u) u+4 \sin (u) u^{2}-2 u^{3}\right) u\right) \\
& \overline{\beta_{1,1}}=\frac{1}{6}\left(\left(-7 u^{3}+18 u\right) \sin (2 u)+\left(-21 u^{2}+6\right) \cos (2 u)-4 u^{4}+12 \cos (u) u^{2}-3 u^{2}-12 \sin (u) u-6\right) \\
& /\left(\left(\left(\frac{1}{2} u^{2}-1\right) \sin (2 u)+u^{3}-2 \sin (u) u^{2}-2 \cos (u) u+\cos (2 u) u+u+2 \sin (u)\right) u\right) \\
& \overline{\beta_{2,1}}=\frac{1}{6}\left(\left(8 u^{3}-12 u\right) \sin (2 u)+u^{4}+10 \sin (u) u^{3}-12 \cos (u) u^{2}+24 \cos (2 u) u^{2}-24 \cos (u)+24\right) \\
& /\left(\left(-4 \sin (u) u^{2}+\sin (2 u) u^{2}+2 u^{3}-4 \cos (u) u+2 \cos (2 u) u+4 \sin (u)-2 \sin (2 u)+2 u\right) u\right) \\
& \overline{\delta_{2,1}}=\frac{1}{6}\left(8 \sin (u) u^{3}+5 \sin (2 u) u^{3}-12 \cos (u) u^{2}-12 \cos (2 u) u^{2}-12 \sin (u) u+6 \sin (2 u) u-48 \cos (u)+12 \cos (2 u)+36\right) \\
& /\left(\left(-2 u-2 \cos (2 u) u-\sin (2 u) u^{2}-4 \sin (u)+2 \sin (2 u)+4 \cos (u) u+4 \sin (u) u^{2}-2 u^{3}\right) u\right) \\
& \overline{\gamma_{2,1}}=\left(\left(-5 u^{2}+18\right) \cos (2 u)-8 \cos (u) u^{2}+u^{2}-16 \sin (u) u+20 \sin (2 u) u-48 \cos (u)+30\right) \\
& /\left(12 \cos (2 u) u^{2}+6\left(2 u^{3}-4 \sin (u) u^{2}+\sin (2 u) u^{2}-4 \cos (u) u+2 u+4 \sin (u)-2 \sin (2 u)\right) u\right) \\
& \left\{\begin{array}{l}
\overline{\beta_{0,1}}=-\frac{49}{160}-\frac{13}{1680} u^{2}-\frac{277}{1728000} u^{4}-\frac{14911}{13970880000} u^{6}+\frac{377539}{4358914560000} u^{8} \\
\overline{\beta_{1,1}}=-\frac{13}{10}+\frac{67}{2100} u^{2}+\frac{289}{378000} u^{4}+\frac{7703}{873180000} u^{6}-\frac{334687}{1362160800000} u^{8} \\
\overline{\beta_{2,1}}=\frac{97}{160}-\frac{29}{1200} u^{2}-\frac{7309}{12096000} u^{4}-\frac{108337}{13970880000} u^{6}+\frac{3467297}{21794572800000} u^{8} \\
\overline{\delta_{2,1}}=-\frac{33}{80}+\frac{23}{1400} u^{2}+\frac{179}{403200} u^{4}+\frac{15571}{2328480000} u^{6}-\frac{263267}{3632428800000} u^{8} \\
\overline{\gamma_{2,1}}=\frac{23}{240}-\frac{1}{2100} u^{2}-\frac{373}{6048000} u^{4}-\frac{15901}{6985440000} u^{6}-\frac{11203}{222393600000} u^{8}
\end{array}\right. \\
& \left(\begin{array}{r}
\beta_{0}=\frac{1}{6}\left(\left(-2 u^{3}+24 u\right) \sin (u)-u^{4}-12 \cos (u) u^{2}+24 \cos (u)-24\right) \\
/\left(\left(-4 \sin (u) u^{2}+\sin (2 u) u^{2}+2 u^{3}-4 \cos (u)+2 \cos (2 u) u+4 \sin (u)-2 \sin (2 u)+2 u\right) u\right)
\end{array}\right. \\
& \beta_{1}=\frac{1}{6}\left(\left(u^{3}-6 u\right) \sin (2 u)+\left(3 u^{2}-6\right) \cos (2 u)+4 u^{4}+12 \cos (u) u^{2}-3 u^{2}-12 \sin (u) u+6\right) \\
& /\left(u\left(\left(\frac{1}{2} u^{2}-1\right) \sin (2 u)+u^{3}-2 \sin (u) u^{2}-2 \cos (u) u+\cos (2 u) u+u+2 \sin (u)\right)\right) \\
& \beta_{2}=\frac{1}{6}\left(22 \sin (u) u^{3}+\sin (2 u) u^{3}-5 u^{4}+36 \cos (u) u^{2}-6 \cos (2 u) u^{2}-24 \sin (u) u-18 u^{2}+24 \cos (u)-12 \cos (2 u)-12\right) \\
& /\left(\left(-2 u-2 \cos (2 u) u-\sin (2 u) u^{2}-4 \sin (u)+2 \sin (2 u)+4 \cos (u) u+4 \sin (u) u^{2}-2 u^{3}\right) u\right) \\
& \delta_{2}=\frac{1}{6}\left(-8 \sin (u) u^{3}+\sin (2 u) u^{3}-12 \cos (u) u^{2}-12 \sin (u) u+6 \sin (2 u) u+12 u^{2}-48 \cos (u)+12 \cos (2 u)+36\right) \\
& /\left(\left(-2 u-2 \cos (2 u) u-\sin (2 u) u^{2}-4 \sin (u)+2 \sin (2 u)+4 \cos (u) u+4 \sin (u) u^{2}-2 u^{3}\right) u\right) \\
& \gamma_{2}=\frac{\frac{1}{6}\left(\left(-u^{2}+6\right) \cos (2 u)+8 \cos (u) u^{2}+5 u^{2}-32 \sin (u) u+4 \sin (2 u) u-48 \cos (u)+42\right)}{\left(\left(-4 \sin (u) u^{2}+\sin (2 u) u^{2}+2 u^{3}-4 \cos (u) u+2 \cos (2 u) u+4 \sin (u)\right)-2 \sin (2 u)+2 u\right) u}
\end{aligned}
$$


$\left\{\begin{array}{l}\beta_{0}=-\frac{1}{160}-\frac{1}{1680} u^{2}-\frac{53}{1728000} u^{4}-\frac{15119}{13970880000} u^{6}-\frac{115693}{4358914560000} u^{8} \\ \beta_{1}=\frac{3}{10}+\frac{1}{700} u^{2}+\frac{41}{378000} u^{4}+\frac{3847}{873180000} u^{6}+\frac{165817}{1362160800000} u^{8} \\ \beta_{2}=\frac{113}{160}-\frac{1}{1200} u^{2}-\frac{941}{12096000} u^{4}-\frac{46433}{13970880000} u^{6}-\frac{2074607}{21794572800000} u^{8} \\ \delta_{2}=\frac{17}{80}+\frac{1}{4200} u^{2}+\frac{19}{403200} u^{4}+\frac{5219}{2328480000} u^{6}+\frac{83119}{1210809600000} u^{8} \\ \gamma_{2}=\frac{7}{240}+\frac{1}{2100} u^{2}+\frac{43}{6048000} u^{4}-\frac{269}{6985440000} u^{6}-\frac{84803}{10897286400000} u^{8}\end{array}\right.$

For $k=3$,

$y_{n}=y_{n+2}+h \overline{\beta_{0,1}(u \cos (u), \sin (u))} f_{n}+h \overline{\beta_{1,1}(\cos (u), \sin (u))} f_{n+1}+h \overline{\beta_{2,1}(\cos (u), \sin (u))} f_{n+2}$

$+h \overline{\beta_{3,1}(\cos (u), \sin (u))} f_{n+3}+h^{2} \overline{\delta_{3,1}(\cos (u), \sin (u))} g_{n+3}+h^{3} \overline{\gamma_{3,1}(\cos (u), \sin (u))} l_{n+3}$

$y_{n+1}=y_{n+2}+h \overline{\beta_{0,2}(\cos (u), \sin (u))} f_{n}+h \overline{\beta_{1,2}(u \cos (u), \sin (u))} f_{n+1}+h \overline{\beta_{2,2}(\cos (u), \sin (u))} f_{n+2}$

$+h \overline{\beta_{3,2}(\cos (u), \sin (u))} f_{n+3}+h^{2} \overline{\delta_{3,2}(\cos (u), \sin (u))} g_{n+3}+h^{3} \overline{\gamma_{3,2}(\cos (u), \sin (u))} l_{n+3}$

$y_{n+3}=y_{n+2}+h \beta_{0}(\cos (u), \sin (u)) f_{n}+h \beta_{1}(\cos (u), \sin (u)) f_{n+1}+h \beta_{2}(u \cos (u), \sin (u)) f_{n+2}$

$+h \beta_{3}(\cos (u), \sin (u)) f_{n+3}+h^{2} \delta_{3}(\cos (u), \sin (u)) g_{n+3}+h^{3} \gamma_{3}(\cos (u), \sin (u)) l_{n+3}$

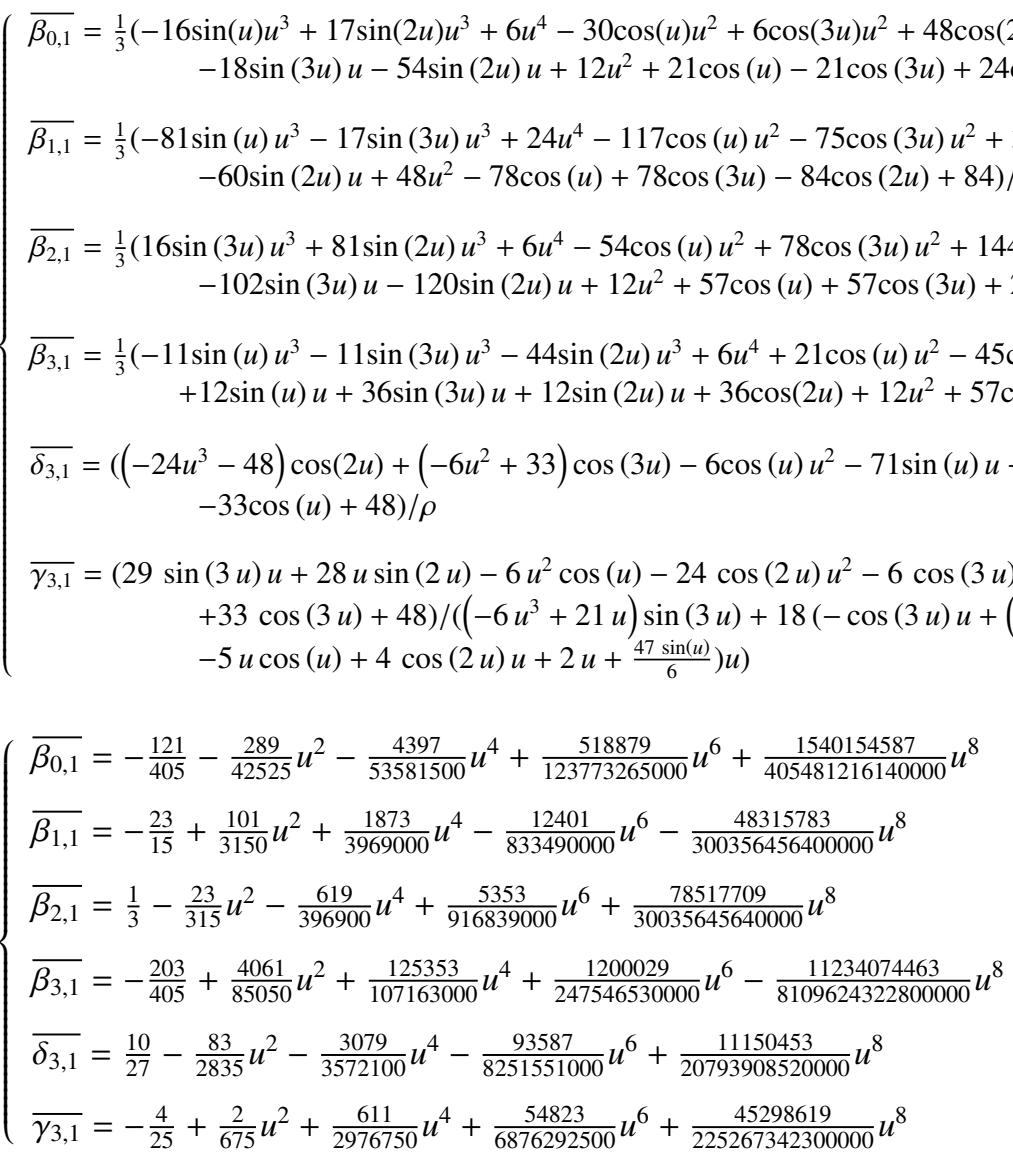




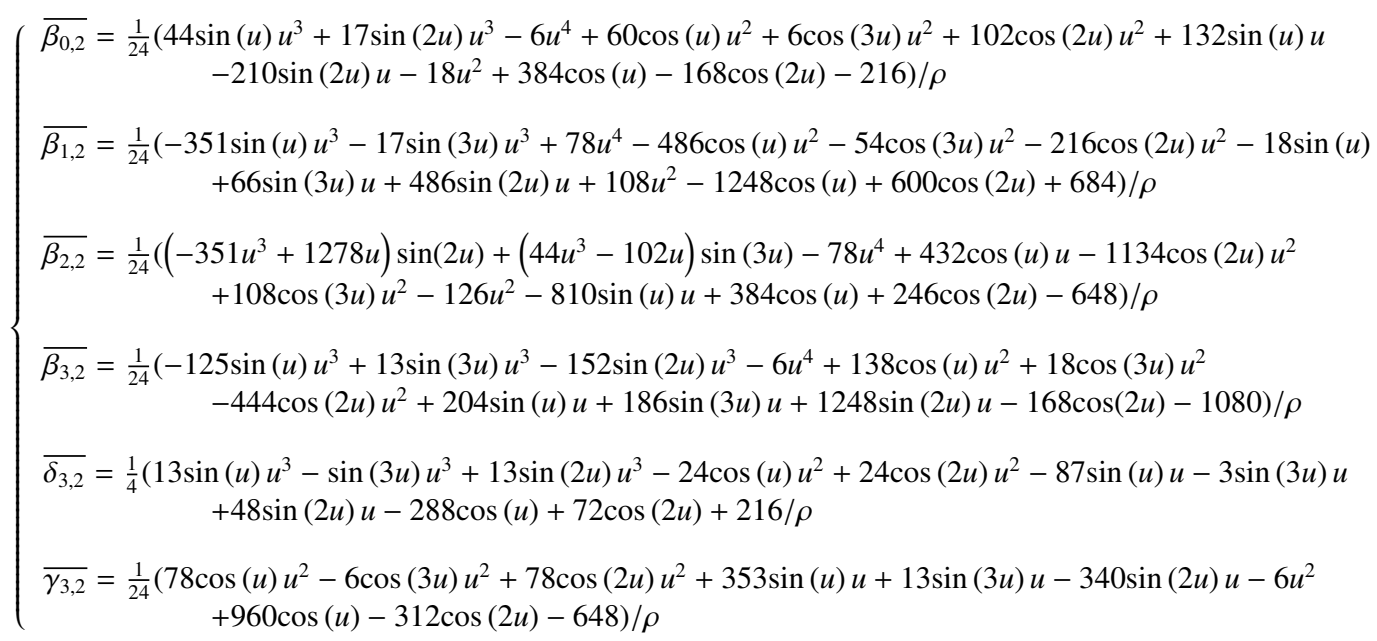

$\left\{\begin{array}{l}\overline{\beta_{0,2}}=\frac{1}{90}+\frac{1}{800} u^{2}+\frac{157}{1984500} u^{4}+\frac{1053089}{293388480000} u^{6}+\frac{1456666699}{1201425825600000} u^{8} \\ \overline{\beta_{1,2}}=-\frac{61}{160}-\frac{331}{67200} u^{2}-\frac{14369}{42336000} u^{4}-\frac{6318043}{391184640000} u^{6}-\frac{611857117}{1067934067200000} u^{8} \\ \overline{\beta_{2,2}}=-1+\frac{19}{3360} u^{2}+\frac{613}{1058400} u^{4}+\frac{631657}{19559232000} u^{6}+\frac{672527}{513429840000} u^{8} \\ \overline{\beta_{3,2}}=\frac{533}{1440}-\frac{19}{9600} u^{2}-\frac{40501}{127008000} u^{4}-\frac{23157647}{1173553920000} u^{6}-\frac{8248324979}{961140660480000} u^{8} \\ \overline{\delta_{3,2}}=-\frac{11}{48}-\frac{1}{2240} u^{2}+\frac{583}{4233600} u^{4}+\frac{420941}{39118464000} u^{6}+\frac{169075937}{320380220160000} u^{8} \\ \overline{\gamma_{3,2}}=\frac{11}{240}+\frac{47}{33600} u^{2}+\frac{703}{21168000} u^{4}+\frac{491}{195592320000} u^{6}-\frac{1081123}{19776556800000} u^{8}\end{array}\right.$

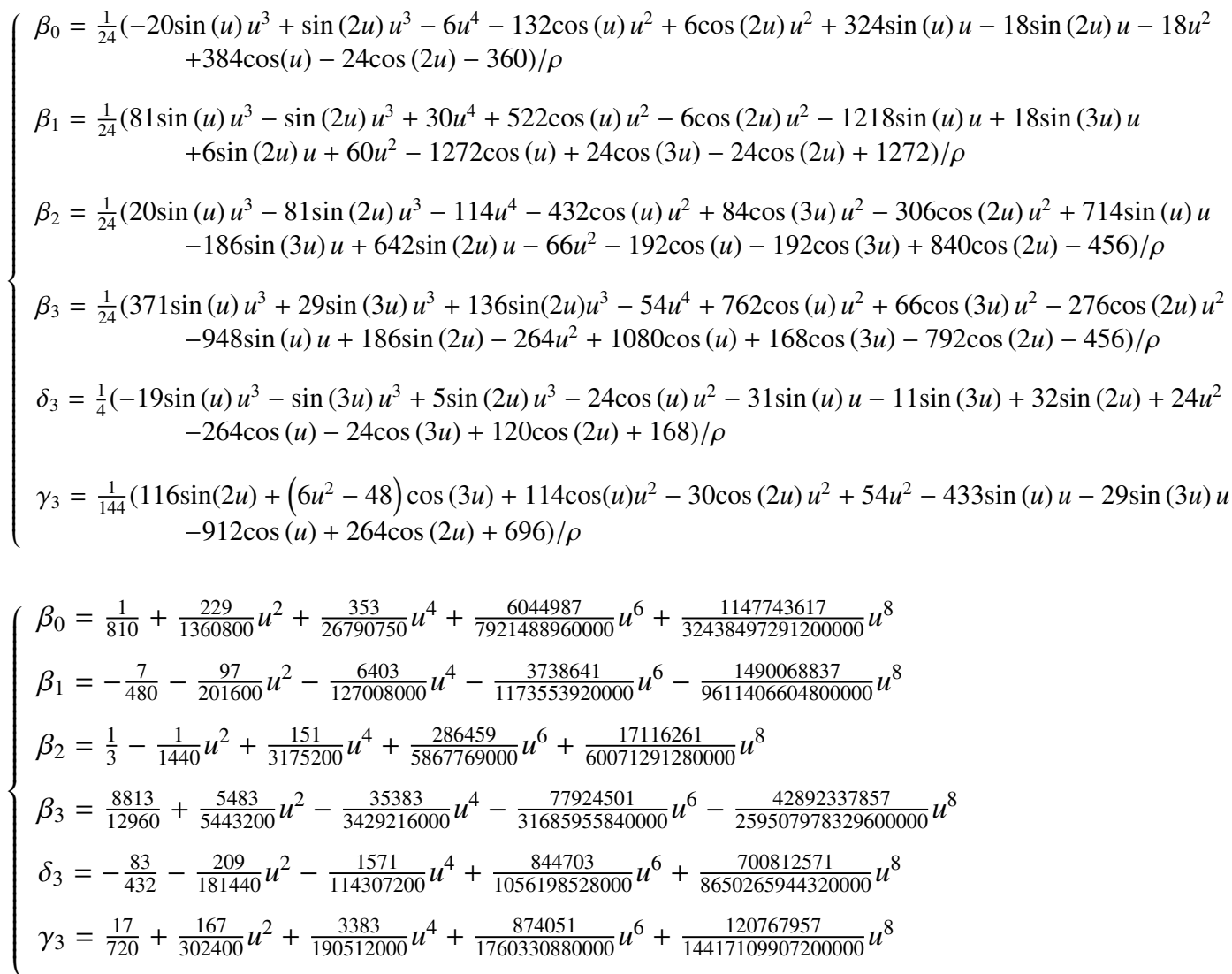


where

$$
\begin{aligned}
\rho= & u\left(18 u^{2} \sin (u)+2 \sin (3 u) u^{2}-9 u^{2} \sin (2 u)\right. \\
& -6 u^{3}+30 u \cos (u)+6 \cos (3 u) u-24 \cos (2 u) u \\
& -47 \sin (u)-7 \sin (3 u)+34 \sin (2 u)-12 u)
\end{aligned}
$$

It is interesting to note that as $u \rightarrow 0$ in the power series expansion of the BTDTFMs, classical third derivative methods based on polynomial basis are recovered.

\section{Basic Properties of BTDTFM}

The basic properties of BTDTFM which includes the Local Truncation Error (LTE), Order, Error Constant, Zero Stability, Convergence and Region of Stability are discussed.

\subsection{Local Truncation Error (LTE) and Order of BTDTFM}

Theorem 1. The BTDTFM has a local truncation error of the form LTE $=C_{k+4} h^{k+4}\left(\omega^{2} y^{k+2}\left(x_{n}\right)-y^{(k+4)}\left(x_{n}\right)\right)+O\left(h^{k+5}\right)$.

\section{Proof 1}

Since the BTDTFM is made up of generalized linear multistep methods with trigonometric coefficients, we associate the BTDTFM with a linear operator $\mathcal{L}_{\omega}\left[y\left(x_{n}\right) ; h\right]$ for the principal method and $\overline{\mathcal{L}_{\omega}}\left[y\left(x_{n}\right) ; h\right]$ for the secondary methods defined respectively by

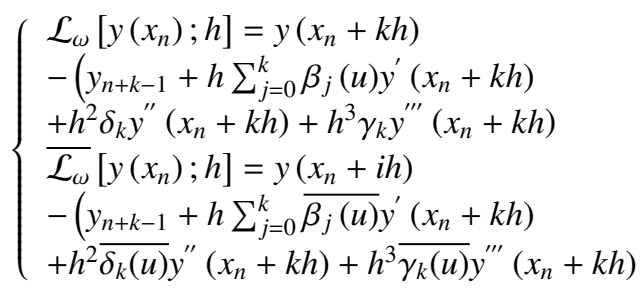

Assuming that $y\left(x_{n}\right)$ is sufficiently differentiable, expanding with Taylor series expansions of $y\left(x_{n}+k h\right), y\left(x_{n}+i h\right), y^{\prime}\left(x_{n}+k h\right)$, $y^{\prime \prime}\left(x_{n}+k h\right)$ and $y^{\prime \prime \prime}\left(x_{n}+k h\right)$ about the point $x_{n}$.

Substituting the coefficients $\beta_{j}(u), \delta_{k}(u), \gamma_{k}(u), \overline{\beta_{j}}(u) \bar{\delta}_{k}(u)$ and $\bar{\gamma}_{k}(u)$ into equations (7) and (8) respectively, after simplification, we obtain the order, the Local Truncation Error as presented in Table 1.

\subsection{Consistency of the BTDTFMs}

Since each BTDTFM is of order $p \geq 5>1$, (for each $k$ )as shown in Table 1, then it is consistent (Lambert [57] and Fatunla [58]).

\subsection{Stability of the BTDTFM}

The combined methods (Principal and Secondary) are assemble in the block form given by

$$
A_{1} Y_{\mu+1}=A_{0} Y_{\mu}+h B_{0} F_{\mu}+h B_{1} F_{\mu+1}+h^{2} G_{\mu+1} C_{1}+h^{3} D_{1} L_{\mu+1}
$$

Where

$$
\begin{aligned}
& Y_{\mu+1}=\left(y_{n+1}, y_{n+2}, y_{n+3}\right)^{T}, Y_{\mu}=\left(y_{n-2}, y_{n-1}, y_{n}\right)^{T}, \\
& F_{\mu+1}=\left(f_{n+1}, f_{n+2}, f_{n+3}\right)^{T},
\end{aligned}
$$

$F_{\mu}=\left(f_{n-2}, f_{n-1}, f_{n}\right)^{T}, G_{\mu+1}=\left(g_{n+1}, g_{n+2}, g_{n+3}\right)^{T}, L_{\mu+1}=\left(l_{n+1}, l_{n+2}, l_{n+3}\right)^{T}$ $A_{0}, A_{1}, B_{0}, B_{1}, C_{1}$ and $D_{1}$ are $k \times k$ matrices defined in canonical form as follows:

For $k=2$, we have

$$
\begin{aligned}
A_{1}=\left(\begin{array}{ll}
-1 & 0 \\
-1 & 1
\end{array}\right), \quad A_{0}=\left(\begin{array}{cc}
0 & -1 \\
0 & 0
\end{array}\right), \quad B_{0}=\left(\begin{array}{cc}
0 & \bar{\beta}_{0,0} \\
0 & \beta_{0}
\end{array}\right), \\
\mathrm{B}_{1}=\left(\begin{array}{cc}
\bar{\beta}_{1,0} & \bar{\beta}_{2,0} \\
\beta_{1} & \beta_{2}
\end{array}\right), \\
C_{1}=\left(\begin{array}{cc}
0 & \bar{\delta}_{2,0} \\
0 & \delta_{2}
\end{array}\right) \text { and } D_{1}=\left(\begin{array}{cc}
0 & \bar{\gamma}_{2,0} \\
0 & \gamma_{2}
\end{array}\right)
\end{aligned}
$$

For $k=3$, we have

$$
\begin{aligned}
A_{1} & =\left[\begin{array}{ccc}
1 & -1 & 0 \\
0 & -1 & 0 \\
0 & -1 & 1
\end{array}\right], A_{0}=\left[\begin{array}{ccc}
0 & 0 & 0 \\
0 & 0 & -1 \\
0 & 0 & 0
\end{array}\right], B_{0}=\left[\begin{array}{ccc}
0 & 0 & \bar{\beta}_{0,1} \\
0 & 0 & \bar{\beta}_{0,0} \\
0 & 0 & \beta_{0}
\end{array}\right], \\
B_{1} & =\left[\begin{array}{ccc}
\bar{\beta}_{1,1} & \bar{\beta}_{2,1} & \bar{\beta}_{3,1} \\
\bar{\beta}_{1,0} & \bar{\beta}_{2,0} & \bar{\beta}_{3,0} \\
\beta_{1} & \beta_{2} & \beta_{3}
\end{array}\right], \\
C_{1} & =\left[\begin{array}{ccc}
0 & 0 & \bar{\delta}_{3,1} \\
0 & 0 & \bar{\delta}_{3,0} \\
0 & 0 & \delta_{3}
\end{array}\right] \text { and } D_{1}=\left[\begin{array}{lll}
0 & 0 & \bar{\gamma}_{3,1} \\
0 & 0 & \bar{\gamma}_{3,0} \\
0 & 0 & \gamma_{3}
\end{array}\right]
\end{aligned}
$$

\subsection{Zero Stability}

Definition 2. A block method is zero stable if the roots of the first characteristic polynomial have modulus less than or equal to one and those of modulus one do not have multiplicity greater than 2. i.e. $\rho(R)=\operatorname{det}\left(R A^{(1)}-A^{(0)}\right)=0$ satisfies $\left|R_{i}\right| \leq 1$ and for those roots with $\left|R_{i}\right|=1$, the multiplicity does not exceed 2 Fatunla [58].

Proposition 3. The BTDTFM is zero stable.

\section{Proof 2}

From the normalized first characteristic polynomial of BTDTFM, we have in canonical form that $\rho_{k}(R)=\operatorname{det}\left[\sum_{i=0}^{1} A_{1-i} R^{i}\right]$. so that $\rho_{k}(R)=0 \Longrightarrow-R^{k-1}(1+R)=0$. Consequently, the roots $R_{j}, j=1,2, \ldots k$ of $\rho_{k}(R)$ satisfy $\left|R_{j}\right|=1$, the roots are simple. Hence for each $k=2$ and $k=3$, the BTDTFM is Zero stable $\square$ 
Table 1: Local Truncation Error of BTDTFM

\begin{tabular}{|c|c|c|c|}
\hline$k$ & LTE & $\operatorname{Order}(p$ & Error Constant $\left(C_{p+1}\right)$ \\
\hline 2 & $\begin{array}{l}\left(-\frac{1}{1800} D^{(6)}(y)(x)-\frac{1}{100} \omega^{2} D^{(4)}(y)(x)\right) h^{6} \\
\left(-\frac{1000}{-200} D^{(6)}(y)(x)-\frac{100}{200} \omega^{2} D^{(4)}(y)(x)\right) h^{6}\end{array}$ & \begin{tabular}{l|l|}
$\mathbf{5}$ \\
$\mathbf{5}$
\end{tabular} & $-\frac{1}{1800} \quad-\frac{1}{200}$ \\
\hline 3 & 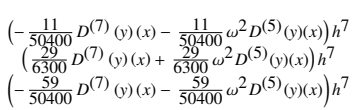 & $\begin{array}{l}6 \\
6 \\
6\end{array}$ & $-\frac{11}{50400} \quad \frac{29}{6300} \quad-\frac{59}{50400}$ \\
\hline
\end{tabular}

\subsection{Convergence of BTDTFM}

Theorem 4. Let $\overline{\mathrm{Y}}$ be an approximation of the solution vector $Y$ for the system obtained from BTDTFM given by equations (6) and (7) respectively, If $e_{n}=\left|y\left(x_{n}\right)-y_{n}\right|$, where the exact solution is several times differentiable on $\left[x_{0}, x_{N}\right]$ and if $\|\mathrm{E}\|=$ $\|\bar{Y}-\mathrm{Y}\|$, then for sufficiently small h,BTDTFM is a $(k+3)$ thorder convergent method. That is $\|\mathrm{E}\|=O\left(h^{k+3}\right)$.

\section{Proof 3}

The proof can be readily obtained, similarly to the one given in Abdulganiy et al. (2017).

\subsection{Linear Stability and Region of Absolute Stability of BT- DTFM}

According to Ndukum et al. [47], linear stability analysis of trigonometrically fitted methods is more complicated than those of the corresponding polynomial-based methods for which $\lambda$ andh occur only in the combination $z=\lambda h$. For trigonometrically fitted methods, three parameters are considered since the step length occurs in both $z=\lambda h$ and $u=\omega h$. To analyze the linear stability of BTDTFM, the block method (25) is applied to the test equations $y^{\prime}=\lambda y, y^{\prime \prime}=\lambda^{2} y$ and $y^{\prime \prime \prime}=\lambda^{3} y$. After simple algebraic simplification and letting $z=\lambda h$, we obtain

$$
Y_{w+1}=M(z, u) Y_{w}
$$

where

$$
\left.M(z, u)=\left[A_{1}-B_{1} z-C_{1} z^{2}-D_{1} z^{3}\right]^{-1}\left[A_{0}+B_{0} z\right)\right]
$$

The rational function $M(z, u)$ is called the amplification matrix which determines the stability of the method.

Definition (Coleman and Ixaru, [59]: A region of stability is a region in the $z u$-plane throughout which $|\rho(z, u)| \leq 1$, where $\rho(z, u)$ is the spectral radius of $M(z, u)$.

Since the stability matrix depends on two parameters $z$ and $u$, we plot the stability regions in the $(z, u)$-plane for both $k=2$ and $k=3$ respectively in Figures 1 and 2 .

Definition (Ndukum etal., [47]): A BTDTFM with variable coefficients $\left(A_{0}(u), A_{1}(u), B_{0}(u)\right.$,

$\left.B_{1}(u), C_{1}(u), D_{1}(u)\right)$ with the stability function $\rho(z, u)$ is said to be A-stable at $u=u_{0}$ if $\left|\rho\left(z, u_{0}\right)\right|<1$ for all $z \in \mathbb{C}^{-}$.

We plot the region $|\rho(z, u)|<1$ (for each $k$ ) in the complex plane via boundary locus method and look for the interval of

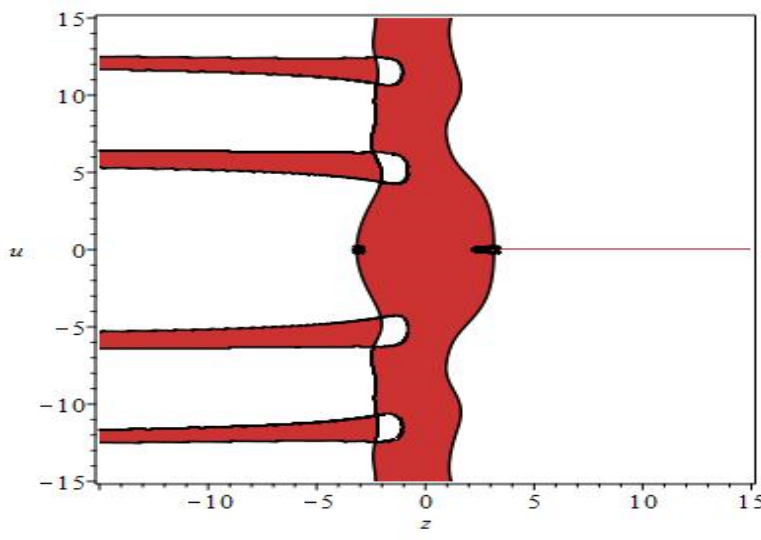

Figure 1: The $z-u$ Plot for BTDTFM $k=2$

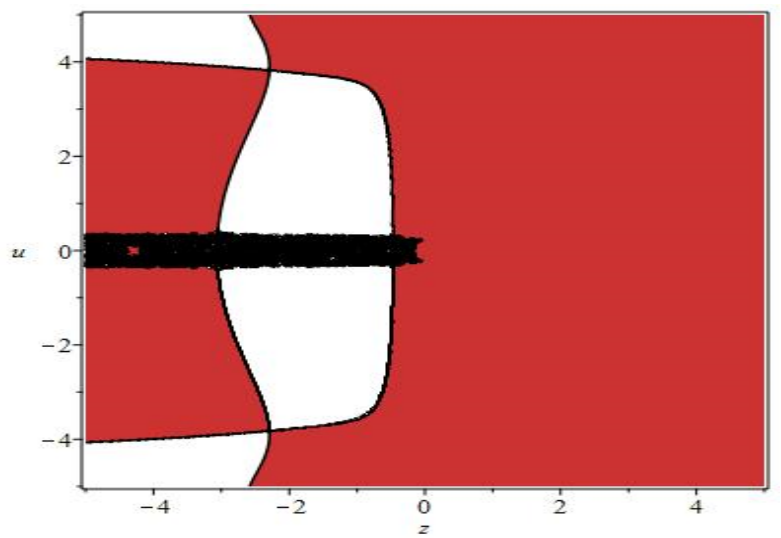

Figure 2: The $z-u$ Plot for BTDTFM $k=3$

$u$ for which $z \in \mathbb{C}^{-}$. For $k=2$, we notice that the values of $u \in[2 \pi, \infty)$ are satisfactory and for $k=3$, the values of $u \in[2.96 \pi, 2.70 \pi]$ are satisfactory. Figures 3 and 4 show the plot of $|\rho(z, u)|<1$ at $u_{0}=2 \pi$ for $k=2$ and $u_{0}=2.96 \pi$ for $k=3$ respectively.

It is also worthy to note that the stability function of the BTDTFM for each $k$ satisfies $|\rho(z, u)|<1$ for all $z \in \mathbb{C}^{-}$, for some $u$ and $\lim _{z \rightarrow \infty} \rho(z, u)=0$ is a property analogous to $L$-stability for the classical methods which is essential for a method to perform well on highly stiff problems (Ndukum etal., [47]). Hence the BTDTFMs are $L-$ stable. 


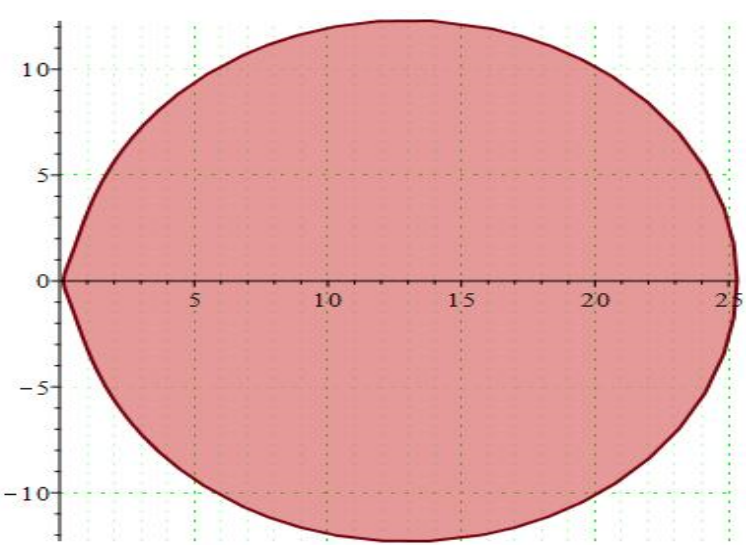

Figure 3: RAS for BTDTFM $k=2$

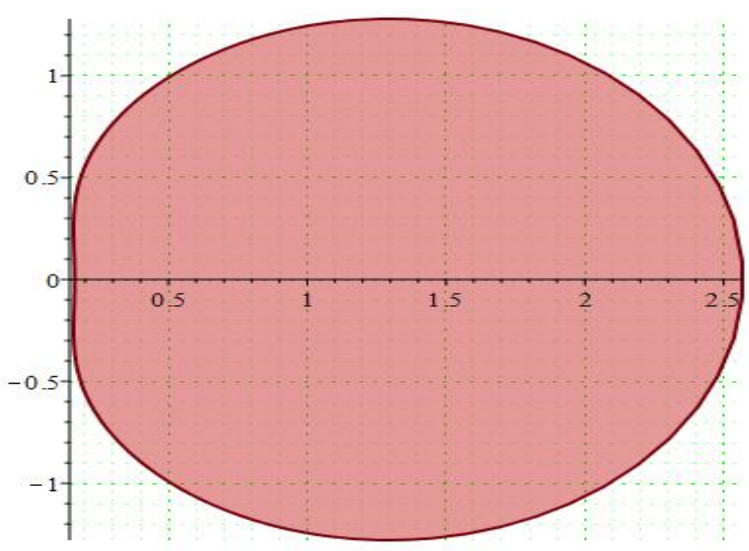

Figure 4: RAS for BTDTFM $k=3$

\section{Estimation of Computational Frequency}

In fitted methods for solving periodic problems, the choice of estimating computational frequency remains a hard nut to crack. A rigorous theory for the exact computation of the frequency is yet to be developed. However, some attempts have been made by a number of researchers. For exponentially fitted methods, the procedure for estimating frequency is given in Ixaru et al. ([29], [60,61]), Vanden Berghe et al. ([30], [62,63]) and Van de Vyve.r [25]. The estimation of the computational frequency of trigonometrically-fitted methods can be found in Ramos and Vigo-Aguiar [64], Vigo-Aguiar and Ramos [65], Ngwane and Jator [42], Jator [48,49] and Ndukum et al., [47]. It is interesting to note that all the aforementioned methods focus on the optimization of the local truncation error (LTE).

In the present study, the approach described in Vanden Berghe et al. [30] for the exponentially fitted methods is adapted to the BTDTFM with Trigonometric coefficients.

It is observed that the LTE of the principal and secondary methods of the BTDTFM consist of a product of three factors. The LTE of the principal method of $\mathrm{k}=2$, for instance, consists of the product of a general factor, a numerical factor in rational form $\left(-\frac{1}{200}\right)$, and a factor which involves two derivatives of the solution vector. For easy reference, we introduce the follow- ing functional $D[y(x) ; \omega]=\left(y^{(6)}(x)+\omega^{2} y^{(4)}(x)\right)$. Assume $\omega$ exists such that $D$ is identically vanishes on the given interval, then the principal method corresponding to that $\omega$ will be exact. The reason according to Vanden Berghe et al. [30] is equivalent to solving the differential equation $y^{(6)}(x)+\omega^{2} y^{(4)}(x)=0$ and, indeed, $\sin (\omega x)$ and $\cos (\omega x)$ are solutions. In general, no constant $\omega$ can be found such that $D$ is identically vanished but it makes logic to discourse the problem of obtaining $\omega$ for which $D$ are kept close to zero as possible for $x$ in the given interval with the result that upon the Taylor series expansion of $\omega^{2}=-\frac{y^{(6)}\left(x_{n}\right)}{y^{(4)}\left(x_{n}\right)}$ about $x_{n}$ given by

$$
D[y(x) ; \omega]=-\left(x-x_{n}\right)\left(y^{(7)}\left(x_{n}\right)-\omega^{2} y^{(5)}\left(x_{n}\right)\right)+O\left(\left(x-x_{n}\right)^{2}\right)
$$

This implies that the bound of $D$ behaves as $h$ and consequently, the bound of LTE for $k=2$ in Table 1 behaves as $h^{7}$ and hence increase the order by 1 .

Remark 1. We remark that other steps and the technical details for implementations are as described in section 3 of Varden Berghe et al. [30].

\section{Numerical Experiments}

A number of numerical examples are provided in this section to illustrate the accuracy and computational efficiency of BTDTFM. We have calculated the absolute error at the end point for stiff problem as $|y(t)-y|$ and the maximum absolute error of the approximate solution as $\operatorname{Err}=\max |y(t)-y|$ for periodic problems and the efficiency is plotted as Number of Function Evaluation (NFE) against $\max |y(t)-y|$.

\subsection{Stiff Problems}

Example 5.1 We consider as our first example a non-linear system of first order differential equations in the range $0 \leq t \leq$ 10

$$
\begin{array}{lrl}
y_{1}^{\prime}=\mu y_{1}+y_{1}^{2} & , y_{1}(0)=-\frac{1}{\mu+2} \\
y_{2}^{\prime}=-y_{2}, & y_{2}(0)=1
\end{array}
$$

whose solution in closed form is given by $y_{1}=-\frac{e^{-2 t}}{\mu+2}, y_{2}=$ $e^{-2 t}$, where $\mu=10000$.

The new BTDTFM is used to solve this problem with $\omega=1$ and compared with the Second Derivative Multistep Method (SDMM) in Hojjati et al. [7], Simpson's $\frac{3}{8}$ Method $\left(S_{\frac{3}{8}}\right)$ in Akinfenwa et al. [16], Multiderivative Hybrid Implicit RungeKutta Method (MHIRK) in Akinfenwa et al. [13], L stable Method (LM) in Mehdizabeh and Molayi [14], Third derivative block hybrid method (TDBHM) in Akinfenwa [50] and the 


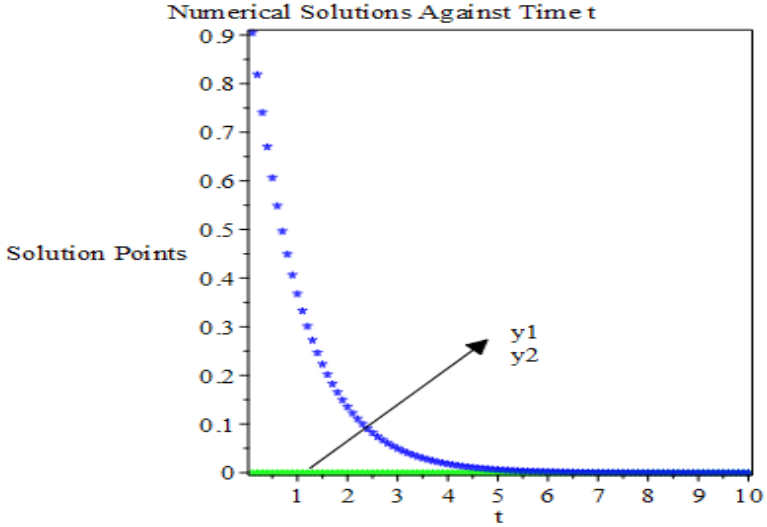

Figure 5: Numerical solution of Example 5.1 for $h=0.1$

results are displayed in Table 2 for the accuracy while Figure 5 show the numerical solution of BTDTFM against the time $t$ for $h=0.1$ and $\omega=1$.

It is evident from Table 2 that the newly constructed method is more accurate than some of the previously known method in the literature. In particular, BTDTFM $k=2$ is far more accurate with large step size compare to SSDM with smaller step size and compete favorably with $S_{\frac{3}{8}}$ with same step size, notwithstanding $S_{\frac{3}{8}}$ is of order 8 .

\section{Example 5.2}

Consider the non-linear stiff system given by

$$
\begin{aligned}
& y_{1}^{\prime}(t)=-1002 y_{1}+1000 y_{2}^{2} \quad, y_{1}(0)=1 \\
& y_{2}^{\prime}(t)=y_{1}-y_{2}\left(1+y_{2}\right) \quad, y_{2}(0)=1
\end{aligned}
$$

Whose exact solution is given as $y_{1}(t)=y_{2}^{2} \quad, \quad y_{2}(t)=e^{-t}$

We solved this problem with $\omega=1$. The results of BTDTFM $k=2$ for this problem in comparison with Exponentially fitted Gauss (EF-Gauss), Gauss-2s , methods in Vaquero and Vigo-Aguiar [18] and Hybrid Backward differentiation formula (HBDF) of Jator and Agyingi [46] for $h=0.1$ and $h=$ 0.01 are displayed in Table 3 . Table 4 presents the results of BTDTFM $k=2$ for different values of $h$ as compared to Extended Continuous Block Backward Differentiation Formula (ECBBDF) of Akinfenwa and Jator [12] and Continuous Block Backward Differentiation Formula (CBBDF) of Akinfenwa et al. [11] for $0 \leq t \leq 10$ while Table 5 shows the results of comparison with the LM method in Mehdizabeh and Molayi [14] and the method in $\mathrm{Wu}$ [6] for $h=0.05$ in the interval $0 \leq t \leq 50$ respectively. The graphical representation of the numerical results of BTDTFM at $h=0.05$ is displayed in Figure 6 .

It is seen from Tables 3-5 that for various values of $h$ considered, BTDTFM does better in terms of accuracy than the methods in [18], [46], [12], [11], [14] and [6] respectively.

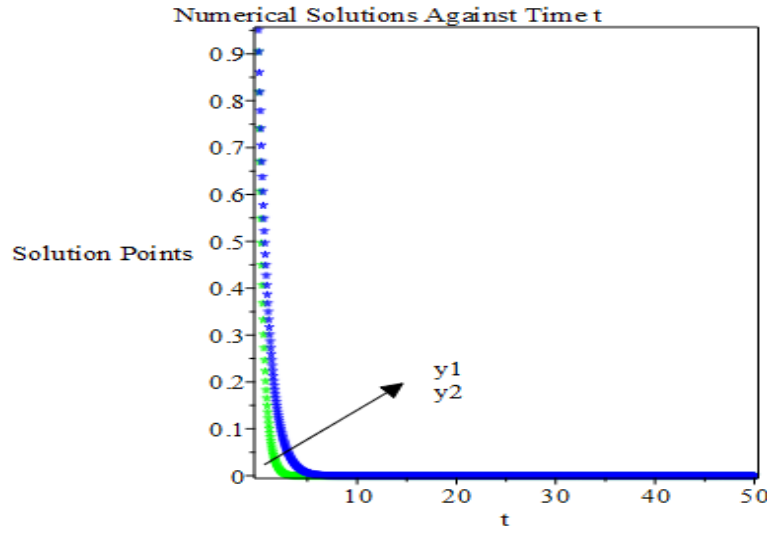

Figure 6: Numerical solution of Example 5.2 for $h=0.05$

\section{Example 5.3}

Consider a classical four-dimensional problem given by

$$
\left[\begin{array}{l}
y_{1}^{\prime}(t) \\
y_{2}^{\prime}(t) \\
y_{3}^{\prime}(t) \\
y_{4}^{\prime}(t)
\end{array}\right]=\left[\begin{array}{c}
-10^{4} y_{1}(t)+100 y_{2}(t)-10 y_{3}(t)+y_{4}(t) \\
-1000 y_{2}(t)+10 y_{3}(t)-10 y_{4}(t) \\
-y_{3}(t)+10 y_{4}(t) \\
-0.1 y_{4}(t)
\end{array}\right],\left[\begin{array}{l}
y_{1}(0) \\
y_{2}(0) \\
y_{3}(0) \\
y_{4}(0)
\end{array}\right]=
$$

$\left[\begin{array}{l}1 \\ 1 \\ 1 \\ 1\end{array}\right]$

The eigenvalue of the Jacobian matrix $\lambda_{1}=-0.1, \lambda_{2}=$ $-1.0, \lambda_{3}=-1000$ and $\lambda_{4}=-10000$. The exact solution of Example 5.3 is given as

$$
\begin{aligned}
& y_{1}(t)=-\frac{89990090}{8999010009} e^{-0.1 t}+\frac{818090}{89901009} e^{-t} \\
& +\frac{9989911}{899010090} e^{-1000 t}+\frac{89071119179}{89990100090} e^{-10000 t} \\
& y_{2}(t)=\frac{9100}{89991} e^{-0.1 t}-\frac{910}{8991} e^{-t}+\frac{9989911}{9989001} e^{-1000 t}, \\
& y_{3}(t)=\frac{100}{9} e^{-0.1 t}-\frac{91}{9} e^{-t}, y_{4}(t)=e^{-1.0 t}
\end{aligned}
$$

This problem is considered to show the performance of BTDTFM on a $4 \times 4$ stiff problems.

Table 6 shows the numerical results of BTDTFM with $\omega=$ 1 in comparison with the Second Derivative Extended Backward Differentiation Formula (SDEBDF) of Ehigie et al. [21] and the method NMTD in Adesanya et al. [22] for $h=0.05$ and 0.1 at $t=20$ while Table 7 displays the results BTDTFM as it compared with AB7 method in Abhulimen and Otunta [19], CEGE method of Abhulimen and Omeike [20] for $h=0.05$ and 0.1 at $t=1$. The graphical representation of the numerical results of BTDTFM at $h=0.05$ is shown in Figure 7.

The results in Tables 6 and 7 showed the superiority of BTDTFM in terms of accuracy over the methods it compared with 
Table 2: Comparison of results for Example 5.1

\begin{tabular}{|c|c|c|c|c|c|c|c|}
\hline & $t$ & $\begin{array}{l}\mathrm{h}=0.0001 \\
\mathrm{SSDM}\end{array}$ & $\mathrm{h}=0.1 S_{\frac{3}{8}}$ & $\begin{array}{l}\mathrm{h}=0.1 \\
M H I R K\end{array}$ & $\begin{array}{l}h=0.0001 \\
L M\end{array}$ & $\begin{array}{l}h=0.1 \\
T D B H M\end{array}$ & $\begin{array}{l}h=0.1 \\
B T D T F M k= \\
2\end{array}$ \\
\hline $\operatorname{Err}\left(y_{1}\right)$ & 3 & $2.48 \mathrm{E}-11$ & $2.03 \mathrm{E}-19$ & $3.06 \mathrm{E}-15$ & $1.78 \mathrm{E}-20$ & $1.34 \mathrm{E}-17$ & $6.54 \mathrm{E}-19$ \\
\hline $\operatorname{Err}\left(y_{2}\right)$ & & $2.47 \mathrm{E}-06$ & $1.44 \mathrm{E}-14$ & $3.08 \mathrm{E}-10$ & $2.08 \mathrm{E}-12$ & $2.98 \mathrm{E}-13$ & $6.56 \mathrm{E}-14$ \\
\hline Err $\left(y_{1}\right)$ & 5 & $3.45 \mathrm{E}-14$ & $1.20 \mathrm{E}-20$ & $9.35 \mathrm{E}-17$ & $2.49 \mathrm{E}-19$ & $2.94 \mathrm{E}-18$ & $2.00 \mathrm{E}-20$ \\
\hline $\operatorname{Err}\left(y_{2}\right)$ & & $2.30 \mathrm{E}-08$ & $3.21 \mathrm{E}-15$ & $6.94 \mathrm{E}-11$ & $4.66 \mathrm{E}-13$ & $1.79 \mathrm{E}-13$ & $1.48 \mathrm{E}-14$ \\
\hline $\operatorname{Err}\left(y_{1}\right)$ & 10 & $3.46 \mathrm{E}-18$ & $1.11 \mathrm{E}-20$ & $8.49 \mathrm{E}-21$ & $5.74 \mathrm{E}-20$ & $3.82 \mathrm{E}-20$ & $1.81 \mathrm{E}-24$ \\
\hline $\operatorname{Err}\left(y_{2}\right)$ & & $3.15 \mathrm{E}-11$ & $4.38 \mathrm{E}-17$ & $9.36 \mathrm{E}-13$ & $6.35 \mathrm{E}-12$ & $2.84 \mathrm{E}-14$ & $2.00 \mathrm{E}-16$ \\
\hline
\end{tabular}

Table 3: Comparison of errors at $t=5$ for Example 5.2

\begin{tabular}{|l|l|l|l|l|}
\hline$h$ & Gauss-2s & EF-Gauss & HBDF & BTDTFM $k=2$ \\
\hline 0.1 & $5.12 E-06$ & $8.36 E-07$ & $5.49 E-11$ & $1.82 E-11$ \\
\hline 0.01 & $6.46 E-12$ & $4.24 E-12$ & $6.07 E-15$ & $2.01 E-16$ \\
\hline \hline
\end{tabular}

Table 4: Comparison of methods at $t=10$ for Example 5.2

\begin{tabular}{|l|l|l|l|l|l|l|}
\hline$h$ & $y$ & $C B B D F_{4}$ & $C B B D F_{5}$ & $E C B B D F_{4}$ & $E C B B D F_{5}$ & BTDTFM $k=2$ \\
\hline 0.02 & $y_{1}$ & $4.88 \mathrm{E}-16$ & $8.37 \mathrm{E}-18$ & $2.48 \mathrm{E}-19$ & $1.33 \mathrm{E}-20$ & $5.76 \mathrm{E}-19$ \\
& $y_{2}$ & $5.39 E-12$ & $9.16 E-14$ & $3.75 E-16$ & $1.35 E-16$ & $6.34 E-15$ \\
\hline 0.01 & $y_{1}$ & $3.13 \mathrm{E}-17$ & $3.39 \mathrm{E}-21$ & $2.68 \mathrm{E}-19$ & $2.87 \mathrm{E}-22$ & $1.82 \mathrm{E}-20$ \\
& $y_{2}$ & $3.45 E-13$ & $1.23 E-17$ & $2.93 E-15$ & $2.93 E-15$ & $2.00 E-16$ \\
\hline
\end{tabular}

Table 5: Comparison of result of at $t=50$ for Example 5.2

\begin{tabular}{|l|l|l|l|l|}
\hline$h$ & $y$ & LMM & WU Method & BTDTFM $k=2$ \\
\hline 0.05 & $y_{1}$ & $4.13 E-25$ & $2.3 E-21$ & $4.89 E-51$ \\
& $y_{2}$ & $1.29 E-22$ & $1.4 E-18$ & $1.27 E-29$ \\
\hline
\end{tabular}

Table 6: Results comparison at $t=20$ for Example 5.3

\begin{tabular}{|l|l|l|l|l|l|}
\hline$h$ & Method & $y_{1}-$ Error & $y_{2}-$ Error & $y_{3}-$ Error & $y_{4}-$ Error \\
\hline 0.05 & SDEBDF & $5.31 \mathrm{E}-12$ & $7.27 \mathrm{E}-11$ & $5.90 \mathrm{E}-09$ & $1.34 \mathrm{E}-09$ \\
& NMTD & - & - & - & - \\
& BTDTFM & $3.71 \times E-17$ & $3.75 E-16$ & $4.13 E-17$ & $3.41 E-15$ \\
& $k=2$ & & & & \\
\hline 0.1 & DEBDF & $2.25 \mathrm{E}-10$ & $2.29 \mathrm{E}-09$ & $2.50 \mathrm{E}-07$ & $2.06 \mathrm{E}-08$ \\
& NMTD & $8.96 \mathrm{E}-07$ & $2.06 \mathrm{E}-08$ & $8.96 \mathrm{E}-10$ & $8.06 \mathrm{E}-11$ \\
& BTDTFM & $1.18 E-15$ & $1.19 E-10$ & $1.31 E-12$ & $1.19 E-13$ \\
& $k=2$ & & & \\
\hline \hline
\end{tabular}

in the reviewed literature.

\subsection{Problems with Periodic Solutions}

\section{Example 5.4}

We consider the following system of couple differential equa- tions which is well known as the two body problem

$$
y_{1}^{\prime \prime}(t)=-\frac{y_{1}}{r^{3}}, \quad y_{1}(0)=1, y_{1}^{\prime}(0)=0
$$

$$
y_{2}^{\prime \prime}(t)=-\frac{y_{2}}{r^{3}}, \quad y_{2}(0)=0, y_{2}^{\prime}(0)=0
$$


Abdulganiy et al. / J. Nig. Soc. Phys. Sci. 2 (2020) 12-25

Table 7: Results comparison at $t=1$ for Example 5.3

\begin{tabular}{|l|l|l|l|l|l|}
\hline$h$ & Method & $y_{1}-$ Error & $y_{2}-$ Error & $y_{3}-$ Error & $y_{4}-$ Error \\
\hline 0.05 & $A B 7$ & $3.2 \mathrm{E}-02$ & $3.2 \mathrm{E}-02$ & $3.3 \mathrm{E}-01$ & $3.7 \mathrm{E}-05$ \\
& $C E G E$ & $3.5 \mathrm{E}-05$ & $3.8 \mathrm{E}-04$ & $3.5 \mathrm{E}-07$ & $3.7 \mathrm{E}-08$ \\
& NMTD & $7.5 \mathrm{E}-11$ & $8.6 \mathrm{E}-10$ & $8.3 \mathrm{E}-08$ & $2.9 \mathrm{E}-09$ \\
& $B T D T F M k=2$ & $2.2 E-12$ & $2.5 E-12$ & $2.5 E-09$ & $1.3 E-11$ \\
\hline 0.1 & $A B 7$ & $2.5 \mathrm{E}-02$ & $2.1 \mathrm{E}-01$ & $2.4 \mathrm{E}-03$ & $2.7 \mathrm{E}-05$ \\
& $C E G E$ & $2.9 \mathrm{E}-05$ & $2.7 \mathrm{E}-04$ & $2.6 \mathrm{E}-06$ & $2.6 \mathrm{E}-08$ \\
& NMTD & $1.4 \mathrm{E}-08$ & $1.3 \mathrm{E}-08$ & $2.1 \mathrm{E}-09$ & $2.7 \mathrm{E}-11$ \\
& BTDTFM $k=2$ & $6.7 E-11$ & $7.5 E-10$ & $7.5 E-08$ & $4.0 E-14$ \\
& & & & & \\
\hline
\end{tabular}

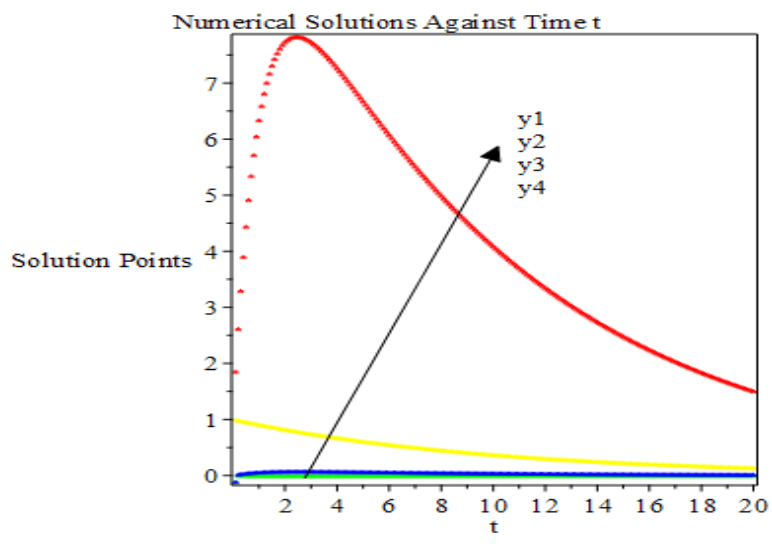

Figure 7: Numerical solution of Example 5.3 for $h=0.05$

where $r=\sqrt{y_{1}^{2}+y_{2}^{2}}$ and whose analytic solution is given by

$$
y_{1}(t)=\cos (t), y_{2}(t)=\sin (t)
$$

The problem was considered in Senu et al. [36] and Abdulganiy et al. [44] in the interval $0 \leq t \leq 10$ with $\omega=1$. The BTDTFM is compared with Block Hybrid Trigonometry Method (BHTM) of Abdulganiy et al. [44] and a New Diagonally Implicit Runge-Kutta Nystrom Method for Periodic IVPs (DIRKNNew) of Senu et al. [36] and the numerical results are displayed in Table 8 below. While the graphical illustration of BTDTFM is shown in Figure 8, Figure 9 shows the efficiency of BTDTFM. It is evident from Table 8 that the newly developed method BTDTFM uses fewer number of function evaluation and consequently produces good results and gives a better approximation when compared with BHTM and DIRKNNew. Also, Figure 9 hows that the method in this paper is efficient.

\section{Example 5.5: Nonlinear Strehmel-Weiner problem}

We consider the nonlinear second order IVP which was also solved by Nguyen et al. [35] and Jator [37] in the interval $0 \leq$ $t \leq 10$ respectively

$$
y_{1}^{\prime \prime}(t)=\left(y_{1}(t)-y_{2}(t)\right)^{3}+6368 y_{1}
$$

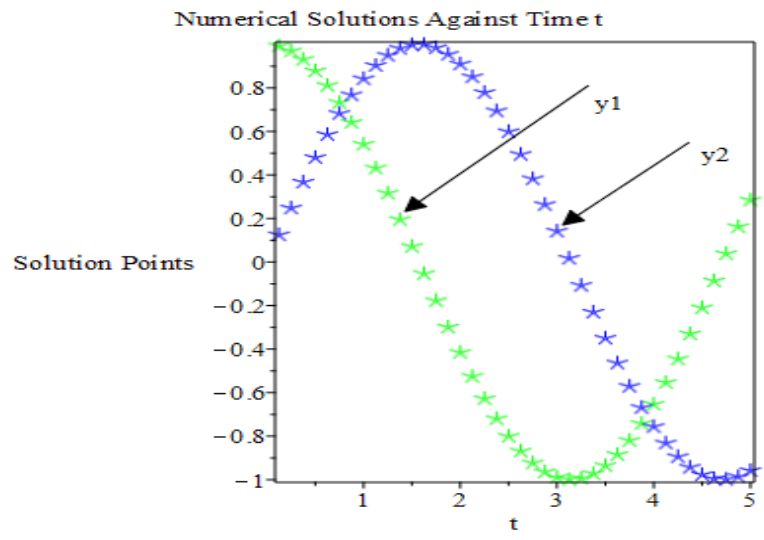

Figure 8: Numerical solution of Example 5.4

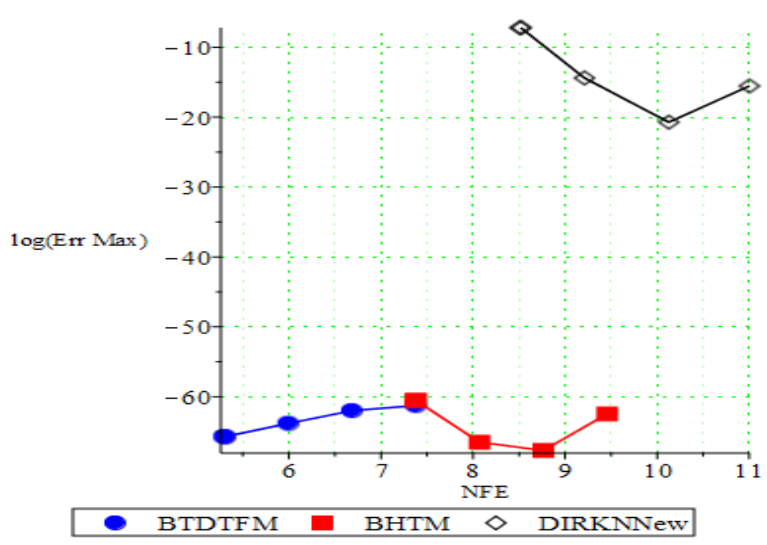

Figure 9: Efficiency Curve of Example 5.4

$$
\begin{aligned}
& \times(t)-6384 y_{2}(t)+42 \cos (10 t), \\
& y_{1}(0)=0.5, y_{1}^{\prime}(0)=0 \\
& y_{1}^{\prime \prime}(t)=-\left(y_{1}(t)-y_{2}(t)\right)^{3}+12768 y_{1}(t)-12784 y_{2}(t) \\
& +42 \cos (10 t), \quad y_{2}(0)=0.5, y_{2}^{\prime}(0)=0
\end{aligned}
$$

with analytic solution in closed form given by $y_{1}(t)=y_{2}(t)=$ $\cos (4 t)-\frac{\cos (10 t)}{2}$.

Numerical results of the maximum global errors of BTDTFM 
Abdulganiy et al. /J. Nig. Soc. Phys. Sci. 2 (2020) 12-25

Table 8: Comparison of Numerical Results for Example 5.4

\begin{tabular}{|c|c|c|c|c|c|c|}
\hline \multirow{2}{*}{$\mathrm{N}$} & \multicolumn{2}{|c|}{ BTDTFM $k=2$} & \multicolumn{2}{|l|}{ BHTM } & \multicolumn{2}{|c|}{ DIRKNNew } \\
\hline & Error & NFE & Error & NFE & Error & NFE \\
\hline 100 & $2.84 E-29$ & 201 & $5.13 E-27$ & 1600 & $7.49 E-4$ & 5000 \\
\hline 200 & $1.92 E-28$ & 401 & $1.30 E-29$ & 3200 & $5.62 E-7$ & 10000 \\
\hline 400 & $1.18 E-27$ & 801 & $4.0 E-30$ & 6400 & $1.00 E-9$ & 25000 \\
\hline 800 & $2.47 E-27$ & 1601 & $7.43 E-28$ & 12800 & $1.78 E-7$ & 60000 \\
\hline
\end{tabular}

Table 9: Comparison of Maximum Errors and Number of Function Evaluation

\begin{tabular}{|l|l|l|l|l|l|}
\hline \multicolumn{4}{|l|}{ BTDTFM $k=2$} & \multicolumn{4}{l|}{ SVBM } & TIRKN 3 \\
\hline$N F E$ & Err & NFE & Err & NFE & Err \\
\hline 751 & $2.25 \mathrm{E}-7$ & 801 & $2.6 \mathrm{E}-7$ & 907 & $2.5 \mathrm{E}-4$ \\
\hline 1126 & $2.95 \mathrm{E}-8$ & 1201 & $1.6 \mathrm{E}-8$ & 1288 & $6.6 \mathrm{E}-6$ \\
\hline 1501 & $7.01 \mathrm{E}-9$ & 1601 & $2.8 \mathrm{E}-9$ & 1682 & $7.0 \mathrm{E}-6$ \\
\hline
\end{tabular}

Table 10: Comparison of Number of steps used in the computation for $\omega=10$

\begin{tabular}{|c|c|c|c|}
\hline Steps & $\begin{array}{c}\text { BTDTFM } k=2 \\
\text { Err }\end{array}$ & $\begin{array}{c}\text { Vigo-Aguiar and Ramos } \\
\text { Err }\end{array}$ & $\begin{array}{c}\text { NEWS5(4)-2 } \\
\text { Err }\end{array}$ \\
\hline 898 & 5.5 & 2.5 & 4.9 \\
\hline 1344 & 6.6 & 3.2 & 6.5 \\
\hline 2123 & 7.8 & 3.4 & 7.8 \\
\hline 2990 & 8.7 & 4.6 & 8.9 \\
\hline 4690 & 9.9 & 5.3 & 9.9 \\
\hline 7215 & 11.0 & 6.1 & 11.0 \\
\hline
\end{tabular}

$k=2$ were compared with Symmetric Boundary Value Method (SBVM) of Jator [37] and Trigonometric Implicit Runge-Kutta Methods (TIRKM) of Nguyen [35] are presented in Table 9 while Figure 10 is the graphical representation of the numerical results of BTDTFM. Also, Figure 11 shows that the method in this paper is more efficient

In Table 9 and Figure 11, we show that a fifth order BTDTFM has almost the same maximum errors with SVBM of order 6 but uses fewer number of function evaluation and consequently a more accurate and more efficient integrator for the nonlinear Strehmel-Weiner problem.

Example 5.6: A Nonlinear Oscillator Lastly, we consider the initial-value problem

$$
\ddot{x}=-100 x+\sin (x), \quad \mathrm{x}(0)=0, \quad \dot{x}(0)=1, \quad t \in[0,20 \pi]
$$

The analytical solution is not known but for comparison purposes we use that $x(20 \pi)=0.000392823991$ given by Tsitouras and Simos [34]. The comparison of different number of steps of BTDTFM $k=2$ with NEW5(4)-2 of Tsitouras and Simos [34] and method in Vigo-Aguiar and Ramos [65] with $\omega=10$. The number of steps are obtained as contained in the references from literature. The error is calculated as $\mathrm{Err}=$

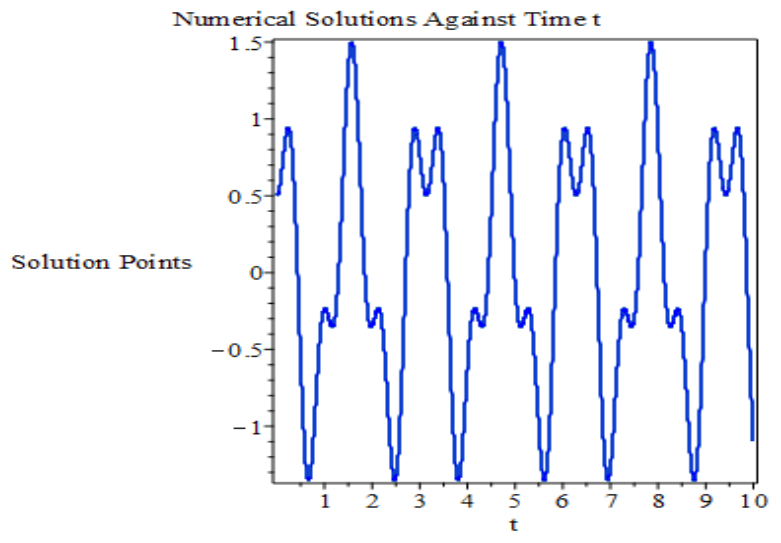

Figure 10: Numerical solution of Example 5.5

$-\log \left(\left|x\left(t_{f}-x_{f}\right)\right|\right)$ where $x_{f}$ is the approximation value obtained at the final point $t_{f}$.

Table 10 revealed that BTDTFM compete favourably with methods in Tsitouras and Simos [34] and method in Vigo-Aguiar and Ramos [65]. 


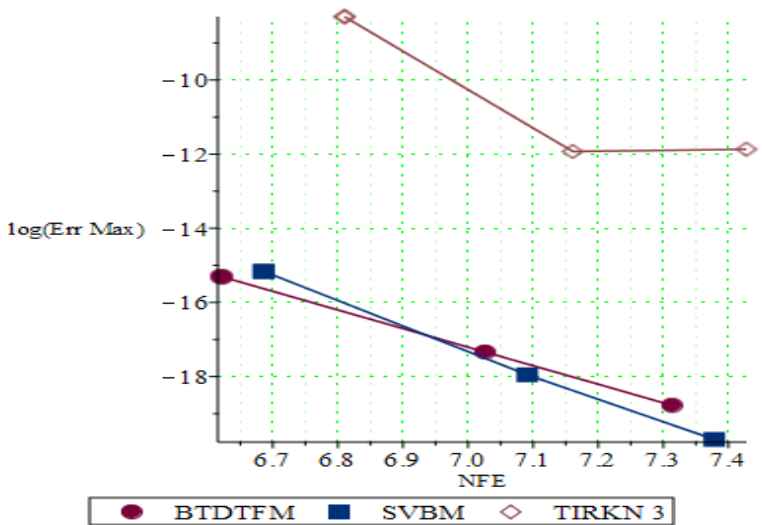

Figure 11: Efficiency Curve of Example 5.5

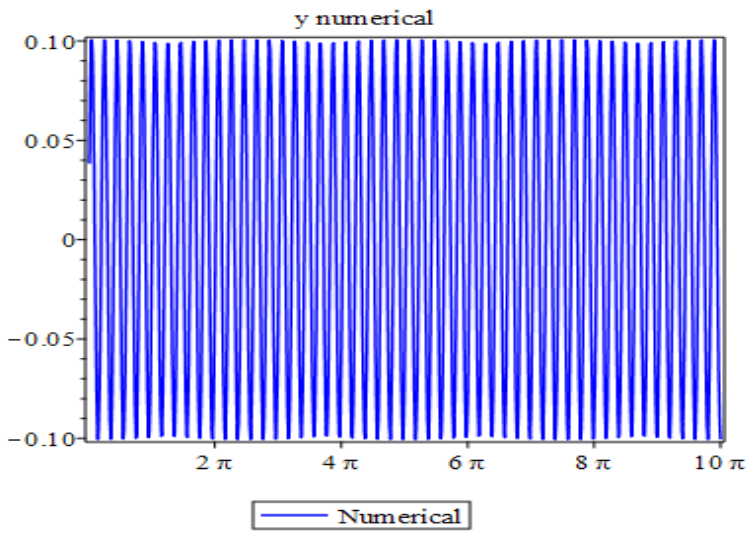

Figure 12: Numerical solution of Example 5.6

\section{Conclusion}

A family of order $k+3$ Block Third Derivative TrigonometricallyFitted Methods are considered and implemented in this paper for Stiff and Periodic Problems. The stability properties of the method was analyzed and was found to be L-stable which in fact is a requirement for an algorithm to solving stiff problems. Numerical results on the representative examples show the accuracy of the BTDTFM on linear and nonlinear stiff problems and its efficiency on nonlinear periodic problems compared to some accurate and efficient methods respectively in the literature. The present study is limited to trigonometric basis. Our future research will consider functionally fitted fitted form as suggested in Nguyen et al. [35].

\section{Acknowledgments}

We thank the referees for the positive enlightening comments and suggestions, which have greatly helped us in making improvements to this paper.

\section{References}

[1] G. G. Dahlquist, A special stability problem for linear multistep methods, BIT 3 (1963) 27.

[2] T. A. Biala S. N. Jator, R. B. Adeniyi, P. L. Ndukum, "Block Hybrid Simpson's Method with Two Offgrid Points for Stiff Systems", International Journal of Nonlinear Science 20 (2015) 3.

[3] C. W. Gear, Hybrid methods for initial value problems in ordinary differential equations. SIAM J. Numer. Anal.2 (1965) 69.

[4] W. H. Enright, "Second Derivative Multistep Methods for Stiff ordinary differential equations", SIAM J. Numer. Anal. 11 (1974) 321.

[5] J. R. Cash, On the Integration of Stiff Systems of ODEs Using Extended Backward Differentiation Formulae. Numerische Mathematik 34 (1980) 235-246.

[6] X. U. Wu. A sixth-order A-stable explicit one-step method for stiff systems, Comput math. Applic.35 (1998) 59.

[7] G. Hojjati, M. Rahimi, S. M. Hosseini. New second derivative multistep methods for stiff systems. Appl. Math. Model 30 (2006) 466.

[8] S. N. Jator, "Solving second order initial value problems by a hybrid multistep method without predictors", Applied Mathematics and Computation 277 (2010) 4036.

[9] R. K Sahi, S. N. Jator, N. A. Khan, A Simpson's-Type Second Derivative Method for Stiff System. International Journal of Pure and Applied Mathematics 81619.

[10] F. F. Ngwane, S. N. Jator, "Block hybrid-second derivative method for stiff systems", Intern. J. Pure Appl. Math. 4 (2012) 543.

[11] O. A. Akinfenwa, S. N. Jator, , N. Yao, Continuous block backward differentiation formula for stiff ordinary differential equations. Computer and Mathematics with Applications 65(2013)996.

[12] O. A. Akinfenwa, S. N. Jator, Extended continuous block backward differentiation formula for stiff systems. Fasciculi Mathematici.(2015). https://doi.org/10.1515/fascmath-2015-0010.

[13] O. A. Akinfenwa, S. A. Okunuga, B. I. Akinnukawe, U. O. Rufai, R. I. Abdulganiy, Multiderivative hybrid implicit Runge-Kutta method for solving Stiff system of a first order Differential equation. Far East Journal of Mathematical Sciences 106(2018)543.

[14] M. Mehdizadeh, M. Molayi, A new class of L-stable hybrid one-step methods for the numerical solution of ordinary differential equations. Journal of Computer Science and Applied Mathematics 1(2015)39.

[15] R. I. Abdulganiy, O. A. Akinfenwa, S. A. Okunuga, A family of $L_{0}$ stable Third derivative Block Methods for solving systems of First Order Initial Value Problems. J. of NAMP 36(2016)47.

[16] O. A. Akinfenwa, R. I. Abdulganiy S. A. Okunuga, V. Irechukwu, Simpson's $\frac{3}{8}$ Type Block Method For Stiff Systems of Ordinary Differential Equations. Journal of the Nigerian Mathematical Society 36(2017)503.

[17] S. A. Okunuga, A fourth order composite two step method for stiff problems. International Journal of Computer Mathematics 72(1999)39.

[18] J. M. Vaquero and J. Vigo-Aguiar, Exponential fitted Runge-Kutta methods of collocation type based on Gauss, Radau, and Labatto traditional methods. Proceedings of the International Conference on Computational and Mathematical Methods in Science and Engineering (CMMSE '07)289.

[19] C. E. Abhulimen, O. F. Otunta, A family of two step Exponentially fitted Multiderivative methods for the numerical integration of stiff IVPs on ODEs. International Journal of Numerical Mathematics 13(2007)1.

[20] C. E. Abhulimen, G. E. Omeike, A Six-Order Exponentially Fitted Scheme for The Numerical Solution of Systems of Ordinary Differential Equations. Journal of Applied Mathematics and Bioinformatics 1(2011)175.

[21] J. O. Ehigie, S. A. Okunuga, A. B. Sofoluwe, A class of exponentiallyFitted second derivative extended backward differentiation formula for solving stiff problems. Fasciculi Mathematici 51(2013)71.

[22] A. O. Adesanya, R. O. Onsachi, , M. R. Odekunle, New algorithm for first order stiff initial value problems. Fasciculi Mathematici 58(2017)2.

[23] D. G. Yakubu, M. Aminu, P. Tumba, M. Abdulhameed, An efficient family of second derivative Runge-Kutta collocation methods for oscillatory systems. Journal of the Mathematical Society 37(2018)111.

[24] G. Vanden Berghe, H. De Meyer, M. Van Daele, T. V. Hecke, Exponentially-Fitted explicit Runge-Kutta methods. Computer Physics Communications 123(1999)7.

[25] H. Van de Vyver, Frequency evaluation for exponentially fitted Runge- 
Kutta methods. Journal of Computational and Applied Mathematics 184(2005)442.

[26] W. Gautschi, Numerical Integration of Ordinary Differential Equations Based on Trigonometric Polynomials, Numerische Mathematik 3(1961)381.

[27] T. Lyche, Chebyshevian Multistep Methods for Ordinary Differential Equations, Numerische Math.19(1972)65.

[28] J. P. Coleman, S. C. Duxbury, Mixed collocation methods for $y^{\prime \prime}=f(x, y)$ . Journal of computational and Applied Mathematics 126(2000)47.

[29] L. Gr. Ixaru, G. Vanden Berghe, H. De Meyer, "Frequency evaluation in exponentially-fitted algorithms for ODEs", Journal of Computational and Applied Mathematics 140 (2002) 423.

[30] G. Vanden Berghe, Ixaru L. Gr., M. Van Daele, Optimal implicit exponentially fitted Runge-Kutta methods. Computer Physics Communications 140 (2001) 346.

[31] G. Vanden Berghe \& M. Van Daele, "Exponentially-fitted Numerov methods", J. Comp. Appl. Math.200(2007) 140.

[32] T. E. Simos, An Exponentially-Fitted Runge-Kutta Method for the Numerical Integration of Initial Value Problems with Periodic or Oscillating Solutions. Computer Physics Communications 115 (1998) 1.

[33] T. E. Simos, Exponentially-Fitted Runge-Kutta-Nyström Method for the Numerical Solution of Initial Value-Problems with Oscillating Solutions. Applied Mathematics Letters 15 (2002) 217.

[34] Ch. Tsitouras, T. E. Simos, Optimized Runge-Kutta pairs for problem with oscillatory solutions, Journal of computational and Applied Mathematics 147 (2002) 397.

[35] H. S. Nguyen, R. B. Sidje, N. H. Cong, Analysis of trigonometric implicit Runge-Kutta methods. Journal of computational and Applied Mathematics 198 (2007) 187.

[36] N. Senu, M. Suleimon, F. Ismail, M. Othman, A New Diagonally Implicit Runge-Kutta-Nystrom Method for Periodic IVPs. WSEAS Transactions on Mathematics 9(2010)679.

[37] S. N. Jator, Trigonometric symmetric boundary value method for oscillating solutions including the sine-Gordon and Poisson equations. Applied \& Interdisciplinary Mathematics 3(2016)1.

[38] F. F. Ngwane, S. N. Jator, Solving Oscillatory Problems Using a Block Hybrid Trigonometrically Fitted Method with Two Off-Step Points. Texas State University. San Marcos, Electronic Journal of Differential Equation 20(2013)119.

[39] F. F. Ngwane, S. N. Jator, Block hybrid method using trigonometric basis for initial problems with oscillating solutions. Numerical Algorithm 63(2013)713.

[40] S. N. Jator, S. Swindell, R. D. French, Trigonometrically Fitted Block Numerov Type Method for $y^{\prime \prime}=f\left(x, y, y^{\prime}\right)$. Numer Algor 62(2013)13.

[41] F. F. Ngwane, S. N. Jator, Trigonometrically-Fitted Second Derivative Method for Oscillatory Problems. Springer Plus 3(2014)304.

[42] F. F. Ngwane, S. N. Jator, A Family of Trigonometrically Fitted Enright Second Derivative Methods for Stiff and Oscillatory Initial Value problems. Journal of Applied Mathematics (2015)1.

[43] R. I. Abdulganiy, O. A. Akinfenwa, S. A. Okunuga, Maximal Order Block Trigonometrically Fitted Scheme for the Numerical Treatment of Second Order Initial Value Problem with Oscillating Solutions. International Journal of Mathematical Analysis and Optimization(2017)168.

[44] R. I. Abdulganiy, O. A. Akinfenwa, S. A. Okunuga, G. O. Oladimeji, A robust block hybrid trigonometry method for the numerical integration of oscillatory second order nonlinear initial value problem. AMSE journals-
AMSE IIETA publication 2017 series 54(2017)497.

[45] R. I. Abdulganiy, O.A. Akinfenwa, S. A. Okunuga, Construction of L stable second derivative trigonometrically fitted block backward differentiation formula for the solution of oscillatory initial value problems, African Journal of Science, Technology, Innovation and Development 10(2018)411.

[46] S. N. Jator and E. Agyingi, Block Hybrid $k$-Step Backward Differentiation Formulas for Large Stiff Systems. International Journal of Computational Mathematics. (2014).https://doi.org/ 10.1155/2014/162103.

[47] P. L. Ndukum, Biala, T. A., S. N. Jator, R. B. Adeniyi, On a family of trigonometrically fitted extended backward differentiation formulas for stiff and oscillatory initial value problems. Numer Algor, https://doi.org/ 10. 1007/s11075-016-0148-1.

[48] S. N. Jator Implicit third derivative Runge-Kutta-Nyström method with trigonometric coefficients. Numerical Algorithms 70(2015)133.

[49] S. N. Jator, Block third derivative method based on trigonometric polynomials for periodic initial-value problems. Afrika Matematika 27 (2016) 365 .

[50] O. Akinfenwa, Third derivative hybrid block integrator for solution of stiff systems of initial value problems. Afrika Matematika 28(2017)629.

[51] W. H. Enright, Continuous numerical methods for ODEs with defect control. Journal of Computational and Applied Mathematics 125(2000)159.

[52] P. Onumanyi, U.W. Sirisena, S. N. Jator, "Continuous finite difference approximations for solving differential equations", International Journal of Computer Mathematics 72 (1999) 15.

[53] W. E. Milne. Numerical Solution of Differential Equations. John Wiley and Sons, New York, NY, USA, 1953.

[54] J. B. Rosser. A Runge-Kutta for all seasons, SIAM Review 9 (1967) 417.

[55] L. F. Shampine, H. A. Watts. Block implicit one-step methods. Math. Comp. 23 (1969) 731.

[56] M. T. Chu, H. Hamilton, Parallel solution of ODE's by multi-block methods. SIAM Journal on Scientific and Statistical Computing 8 (1987) 342.

[57] J. D. Lambert. Computational methods in ordinary differential equations, John Wiley, New York, 1973.

[58] S. O. Fatunla, Numerical methods for initial value problems in ordinary differential equation. United Kingdom: Academic Press Inc, (1988).

[59] J. P. Coleman, L. Gr. Ixaru, P-Stability and Exponential Fitting Methods for $y^{\prime \prime}=f(x, y)$, IMAJ. Num. An. 16 (1996) 179.

[60] L. Gr. Ixaru, M. Rizea, H. De Meyer \& G. Vanden Berghe, "Weights of the exponential fitting multistep algorithms for ODEs", Journal of Computational and Applied Mathematics 132 (2001) 83.

[61] L. Gr. Ixaru, G. Vanden Berghe, H. De Meyer, "Exponentially-fitted Variable two step BDF algorithms for first order ODEs", Computer Physics Communication 150 (2003) 116.

[62] G. Vanden Berghe, H. De Meyer, M. Van Daele \& T. Van Hecke, "Exponentially fitted Runge-Kutta methods", Journal of Computational and Applied Mathematics 125 (2000) 107.

[63] G. Vanden Berghe, L. Gr. Ixaru \& H. De Meyer, "Frequency Determination and Step-length Control for Exponentially-Fitted Runge-Kutta Methods", Journal of Computational and Applied Mathematics 132 (2001) 95.

[64] H. Ramos, "J. Vigo-Aguiar, On the frequency choice in trigonometrically fitted methods", Applied Mathematics Letters 23 (2010) 1378.

[65] J. Vigo-Aguiar \& H. Ramos, "On the choice of the frequency in trigonometrically fitted methods for periodic problems", Journal of computational and Applied Mathematics 277 (2015) 94. 